\section{PSICOLOGÍA IBEROAMERICANA}

\section{Psicología Iberoamericana}

ISSN: 1405-0943

psicología.iberoamericana@uia.mx

Universidad Iberoamericana, Ciudad de

México

México

Fleming-Holland Rutherford, Ander

Reflexiones sobre la Muerte: el Duelo Infantil y el Suicidio Juvenil

Psicología Iberoamericana, vol. 16, núm. 1, junio, 2008, pp. 8-14

Universidad Iberoamericana, Ciudad de México

Distrito Federal, México

Disponible en: http://www.redalyc.org/articulo.oa?id=133915922002

- Cómo citar el artículo

- Número completo

- Más información del artículo

Página de la revista en redalyc.org

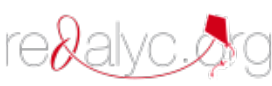

Sistema de Información Científica

Red de Revistas Científicas de América Latina, el Caribe, España y Portugal

Proyecto académico sin fines de lucro, desarrollado bajo la iniciativa de acceso abierto 


\title{
Reflexiones sobre la Muerte: el Duelo Infantil y el Suicidio Juvenil ${ }^{1}$ Reflexions on Death: Childhood Grief and Teenage Suicide
}

\author{
Ander Fleming-Holland Rutherford \\ Facultad de Psicología-Xalapa \\ UNIVERSIDAD VERACRUZANA
}

\section{Resumen}

En este capítulo se examinan las teorías de la muerte y el dolor tanático desde hace cincuenta años hasta el presente, y considera las necesidades especiales del duelo infantil. Se enfatiza la necesidad de explicar la muerte de manera honesta y congruente al nivel del desarrollo de cada niño. El suicidio en los jóvenes se considera del contexto familiar, y se sugieren ciertos lineamientos para que padres de familia prevengan el suicidio en jóvenes. Se concluye que el duelo es un estado natural y necesario después de sufrir una pérdida, y como psicoterapeutas y miembros de la comunidad se debe dejar que se exprese sin minimizarlo ni suprimirlo.

Descriptores: muerte, duelo, suicidio juvenil, niñez, adolescencia.

\begin{abstract}
Theories on death and grieving for the last fifty years are examined as well as the special needs of childhood grieving. The necessity of explaining death honestly and appropriately to the developmental level of each child is emphasized. Teenage suicide is considered in the context of the family, and suggestions are offered for parents to prevent teenage suicide. The author concludes that grieving is a natural and necessary process after suffering a loss, and that psychotherapists as well as the community should allow its expression without minimizing it.
\end{abstract}

Key words: death, grieving, teenage suicide, childhood, adolescence.

\section{Consideraciones teóricas sobre la muerte y el dolor tanático}

Si pretendes soportar la vida, adáptate a la muerte SigMund Freud

Morir es la condición humana y la reflexión referente a la muerte existe en todas las culturas. Desde el comienzo de la historia escrita, la realización de la finitud de la vida ha sido una fuerza poderosa e influyente sobre el hombre. Varios filósofos distinguidos, desde las épocas remotas de la civilización, han señalado que una de las características que más distinguen a la raza humana de otras especies es su capacidad de entender los conceptos de un futuro e, inevitablemente, de una muerte personal.
Pero desde el siglo xVII, el pensamiento filosófico occidental cambió su enfoque desde el campo teológico hacia el campo científico, dando un nuevo énfasis a la maestría científica de la naturaleza, en vez de una maestría espiritual de la persona sobre sí mismo (Toynbee, 1968). Una consecuencia mayor de este cambio tan radical fue un empobrecimiento de las creencias espirituales profundas, sostén filosófico y religioso que ayudaba al hombre a trascender su muerte personal. El concepto de muerte llegó a ser más impermeable hasta que se formó una barrera, en vez de un portón y empezaron los tabúes “civilizados" acerca de los procesos de la muerte y del duelo. La muerte y sus concomitantes se aislaron descontaminados, esterilizados de sus cualidades ritualísticas vivenciales, y sólo así fueron permitidos para entrar a la

${ }^{1}$ Andrée Fleming-Holland Rutherford, Facultad de Psicología-Xalapa, Universidad Veracruzana. Ruiz Cortines No. 300, Col. Belisario Domínguez, C.P. 91079, Xalapa, Veracruz. E-mail: flemingholland@yahoo.com. Tel-fax: 01 (228) 817-86-28 
empeñosa sociedad humana que existía al comienzo de la era despersonalizada de la tecnología.

Las consecuencias sociales de esta dominación filosófica del modelo mecanicista del ser humano, basado en los escritos de Hobbes y Locke aún antes de Descartes (Russell, 1961/2000), han sido un aumento de la fragmentación de la familia y del barrio, lo que los antropólogos nombran grupos de "parentesco", es decir, homogéneos y constantes. Estos valores tradicionales proveían los soportes emocionales y sociales para amortiguar el impacto de la muerte cuando se interponía en la vida. Con la erosión de estos sostenes, el ser humano empezó a distanciarse de la muerte. Abrigado en el misterio y el silencio, sus cualidades desconocidas aumentaron hasta que la muerte personal llegó a ser provincia del profesionista, campo del técnico y terreno cercado con prohibiciones (Kübler-Ross, 1969).

Esta repugnancia cultural por la muerte siguió hasta la Segunda Guerra Mundial, cuando el positivismo lógico empezó a ser derrotado bajo las influencias humanísticas y existencialistas que empezaron a florecer en Europa después de la guerra.

Las dos décadas sucesivas se caracterizaron con la introducción de cursos y talleres sobre la muerte y el proceso de morir en varias universidades y escuelas profesionales europeas y norteamericanas. A la vez, varios académicos como Kastenbaum y Costa (1977) y Kübler-Ross (1969, 1982), entre otros, empezaron a publicar sobre el tema de la muerte. Hoy en día existen revistas profesionales como Omega, Death Studies y el Journal of Thanatology, y varias asociaciones dedicadas específicamente a este tema. Aquí cabría hacer una pregunta: ¿Cuáles descubrimientos empíricos y percepciones psicológicas han resultado de estos trabajos y grupos tanatológicos en las últimas cinco décadas?

Primero que nada, que la muerte es un fenómeno de todas las estaciones de la vida humana. Esto quiere decir que su fuerza directiva está presente en todos nosotros: sanos y enfermos, jóvenes y viejos. No es un tema solamente apropiado a los soldados, moribundos, ancianos o suicidas. Es un ingrediente importante de la vida a través de toda la vida. Así, no beneficiamos a los niños cuando intentamos protegerlos de la experiencia de la muerte de un ser querido. Al contrario, impedimos su desarrollo emocional. Estamos descubriendo que los niños son más capaces de soportar la tensión asociada con la muerte que la tensión asociada con el misterio y el abandono implicados en el resultante vacío silencioso.

En segundo lugar, que el miedo a la muerte puede ser una ocurrencia del segundo orden, reflejando el desplazamiento de una ansiedad de separación; ésta es la posición ortodoxa tradicional del psicoanálisis. Investigaciones con pacientes moribundos geriátricos y psicóticos han indicado que lo opuesto va más al grano; la aprehensión y el miedo referente a la muerte y al proceso de morir pueden por sí mismo producir síntomas psíquicos tales como depresión, miedos exagerados de pérdida, fobias, síntomas físicos tales como insomnio y manifestaciones psicosomáticas e incluso psicóticas (Whal, 1959).

El miedo tanático no suele ser una variable unidimensional, unipolar y unitaria; presenta varios componentes tales como la pérdida de identidad, la soledad o el miedo de "irse al infierno". Así, las connotaciones negativas de la muerte suelen ser asociadas con sentimientos de desarraigo y el tener que enfrentar lo "desconocido" sin la maestría adecuada. Estos sentimientos, al parecer, son más importantes que los de no haber vivido suficientemente o de que la familia sufriría. Suele ser que la actitud hacia la muerte ya no refleja la posibilidad de expiación y salvación consecuente sino aislamiento y pérdida del self (Feifel y Branscomb, 1973; García-Alandete y Gallego-Pérez, 2003; Berti y Scheider-Berti, 2003).

La muerte humana opera simultáneamente entre varios niveles de realidad o significancia, cada uno de los cuales puede funcionar con autonomía. Por ejemplo, existen discrepancias entre los mecanismos conscientes e inconscientes de la muerte. Mientras el miedo tanático al nivel consciente no se destaca verbalmente, al nivel de la fantasía se presenta como una ambivalencia en las imágenes manifestadas, y suele ser totalmente negativo (la "negación") a nivel inconsciente. Este balance contradictorio de aceptación y evitación de la muerte personal parece servir a necesidades poderosas de adaptación. Estos hallazgos indican la necesidad de no aceptar evidencias superficiales del grado de miedo tanático como se afirman conscientemente, dado que existen evidencias contradictorias en otros niveles (Feifel, 1977).

Del mismo modo a cómo se enfrenta una enfermedad grave, la amenaza de la muerte varía mucho entre culturas y situaciones. Por ejemplo, los pacientes geriátricos difieren significativamente de los pacientes con cáncer o con problemas cardíacos en la manera en que manejan situaciones tensionales como competencia, discordia, pérdidas económicas o del empleo, y el proceso de hacer decisiones en general. En contraste con mucha de la literatura clínica tradicional, la cual insiste en que los mecanismos de adaptación utilizados para enfrentar la amenaza de la muerte son los mismos mecanismos, aunque intensificados, que el individuo utiliza en situaciones personales en su vida cotidiana, las investigaciones de Feifel han demostrado repetidamente 
que las estrategias utilizadas para enfrentar a la muerte reflejan modificaciones significativas en la configuración de los mecanismos tradicionales (Feifel y Nagy, 1986; Feifel y Stack, 1989).

¿Varían estos mecanismos desde la niñez hasta la madurez? El nivel de desarrollo cognoscitivo de los niños implica que hay una aproximación diferente a la muerte y al dolor tanático en ellos.

\section{El duelo infantil}

\section{Padre muere, hijo muere, nieto muere \\ Proverbio Zen Budista}

¿Por qué ya no puede volar el pájaro? y ¿cómo llega mi abuelo al cielo si está encerrado en una caja debajo de la tierra? Son preguntas que padres y maestros han oído con mucha frecuencia. La muerte, sin embargo, es un tema muy difícil de manejar por los prejuicios y actitudes culturales (Fleming-Holland, 1991, 1998; García-Alandete y Gallego-Pérez, 2003). Así, es útil saber algo sobre cómo piensan y sienten los niños sobre la muerte y las diferentes etapas de la vida.

Los conceptos infantiles revelan un patrón de desarrollo similar en todos los niños, pero varían ampliamente según la edad. En su desarrollo cognoscitivo, los conceptos progresan de lo concreto a lo abstracto, de vagos a precisos, de inexactos a definidos, de generales a específicos. Incluso muchas pruebas de la inteligencia infantil se basan en esta progresión y acumulación de detalles, por ejemplo, las de Goodenough, Raven, Stanford-Binet, wPPSI y wISC, entre otros (Fleming-Holland, 2000a, 2000b).

Algunos investigadores (p.e.: Hurlock, 1956; KüblerRoss, 1993; Nagy, 1953) hasta hace 15 años, estaban de acuerdo que los conceptos de la vida y la muerte evolucionan en etapas relacionadas con la edad del niño. Así, antes de los cinco años, el niño cree que todos los objetos tienen las mismas cualidades vitales como el ser humano; este animismo es responsable de muchos conceptos erróneos que tienen los niños en esta edad, por ejemplo, que un tren corre porque tiene vida. Entonces los niños no entienden la muerte y no la ven ni como oposición ni como finalización de la vida; más bien, según la teoría tradicional del desarrollo, la ven como un cambio de vivienda o como una salida, no como un proceso final en su pensamiento animístico que inventa cualidades de vida en todo, incluso en cosas inanimadas.
El niño pequeño tiene gran curiosidad tanto acerca del universo externo como acerca del mundo en que vive. Por ende, cuándo y a qué grado va a aparecer el interés en la muerte, lo funerario y qué pasa después de ella, dependerá de las influencias ambientales. Por ejemplo, cuando la muerte ocurre en una persona cercana a la vida de un niño, el impacto será más fuerte en este caso que si es de una persona menos cercana. Según un estudio de Schilder y Wechsler (1934), el niño ve a la muerte de manera impersonal y objetiva, como algo que pasa a otros sin relación a él mismo. Dado que su habilidad para comprender las significancias está todavía limitada, el niño por lo general no se interesa en su propia muerte. El estudio más citado, hecho por Nagy (1953), aclara cómo los conceptos infantiles de la muerte se desarrollan según un patrón cronológico. Aunque la rigidez de las edades se ha modificado recientemente a favor de etapas cognoscitivas (Peterson y Seligman, 2004), es indispensable detallar estos datos, dado que la mayoría de los psicólogos del desarrollo infantil los manejan como si fueran todavía canónicos.

Así, según la teoría clásica, entre los 3 y los 5 años, el niño niega a la muerte como un proceso final. Atribuye la vida y la conciencia al muerto, creyendo que solamente duerme. A esta edad empiezan las preguntas referentes a dónde y cómo la persona sigue viviendo después de la muerte: podría estar confundido al tratar de saber cómo puede moverse una persona en un ataúd lo suficiente para "irse con Dios". Aproximadamente a la edad en que ingresan a la primaria, empiezan a pensar que la muerte es gradual pero temporal; no lo contempla todavía como una finalidad. Hasta la edad de 9 años, la muerte se personifica como una persona aparte, una persona identificada con el proceso, por ejemplo, "la calaca”. Solamente después empieza el niño a reconocer la muerte como un proceso inevitable que es la finalidad de la vida (Hurlock, 1956).

Hallazgos recientes en el campo de la neurobiología interpersonal reafirman la teoría clásica del desarrollo (Siegel, 1999, 2006). Sostiene Siegel que la mente, a través de la retroalimentación social, forma nuevas veredas neuronales en el cerebro, las cuales reestructuran la conducta futura. Así, el modelo paterno cobra aún más sostén teórico, dado que este investigador, entre otros, está comprobando a nivel micro, demostrable, lo afirmado en la teoría clásica: los patrones emocionales y conductuales son aprendidos.

El estudio más completo, y el más citado hasta la fecha sobre los conceptos de la muerte en los niños fue el de Markusen y Fulten (1991), en donde se analizaron 378 dibujos con entrevistas sobre niños húngaros de 3 
a 10 años. Resultaron tres etapas principales de desarrollo cognoscitivo referente a la muerte. En la primera etapa, hasta la edad de los 5 años, el niño no concibe la muerte como un evento final; en esta etapa los niños ven al muerto como "menos vivo" y con posibilidades de revivir después. El aspecto que más se comprende en esta etapa es la separación. En la segunda etapa, los niños empiezan a pensar en la muerte como final pero sigue una creencia que se la puede evitar, uno le puede ganar a "la calaca". Es decir, en esta etapa entre los 6 y los 9 años, se puede notar una tendencia marcada para personificar a la muerte. La tercera etapa, que empieza entre los 9 y los 10 años, se caracteriza por la aceptación de la muerte como final inevitable, y resulta una extensión de una personalidad personal y propia (Markusen y Fulton, 1971).

Las consecuencias conductuales de estas investigaciones son múltiples y siempre reflejan su estructura cognoscitiva. A veces, los adultos interpretan las reacciones infantiles como insensibles, dado que el niño no puede mostrar ni congoja ni remordimiento hasta que posee las estructuras cognoscitivas para ordenar memorias del pasado y anticipar su futuro, las cuales se desarrollan hasta la etapa de operaciones formales. Pero los niños muestran su duelo en otras formas: enojo, ansiedad, enuresis y otros problemas conductuales (Markusen y Fulton, 1971).

Es necesario hablar de la muerte a los niños en dos circunstancias diversas. Primero, el niño puede haber sufrido la pérdida de un ser querido o de un conocido casual. Y segundo, el niño puede tener curiosidad acerca de la muerte por cualquier razón. La diferencia entre las dos circunstancias es el estado emocional de los adultos y los niños involucrados. ¿Cómo explicar la muerte a un niño? Depende de su etapa cognoscitiva, en la intensidad y rango de su interés, en la clase de preguntas que hace y en el clima emocional alrededor del niño. Ayudar a un niño a enfrentar la muerte es difícil, y dado que cada niño lo enfrenta de manera única, no se pude pretender que existan reglas generales (Kübler-Ross, 1993; Siegel y Hartzell, 2004). Sin embargo, sí existen unas cuantas reglas de salud emocional aplicables al duelo o la curiosidad infantil por la muerte:

1) Siempre contestar cualquier pregunta clara y verdaderamente, de manera tan directa como se pueda. No se deben ofrecer más detalles de lo que pide el niño; si quiere saber más, él preguntará.

2) No se evite el tema de la muerte ni se evite asistir a una funeraria. La literatura (Kübler-Ross, 1993) está de acuerdo en que a los niños se les debe permitir asistir a un velorio y/o cementerio, para realizar la finalidad de la muerte, siempre y cuando vayan con adultos de confianza.

3) No deben cerrarse las puertas a dudas, preguntas y diferencias de opinión. Hay que respetar la personalidad única de la otra persona, aunque sea un niño.

4) No desanimar la expresión emocional de congoja: enojo, llanto, culpabilidad, desesperación y protesta; son reacciones naturales a la desorganización familiar.

5) No hay que decir al niño cosas que luego tendrá que desaprender; hay que evitar cuentos de hadas, medias verdades o enredos. Evitar también el lenguaje figurativo porque el niño entiende de forma literal. La honestidad es el único camino que se debe tomar.

6) No legislar sus propias convicciones, evite abstracciones; los pensamientos deben traducirse al lenguaje y comprensión del niño, según su nivel de desarrollo cognoscitivo.

7) No tener miedo a expresar las propias emociones. Hay que ser humano, pero intentando evitar muestras de ansiedad, ser breve al asignar la tarea de informar a alguien menos involucrado emocionalmente sobre el hecho.

8) Hablar a las emociones del niño y no a su intelecto. El niño percibe el clima emocional y se da cuenta cuando la familia está en crisis. El niño necesita saber que aunque los adultos estén tristes ahora, esto pasará. Necesita escuchar que los adultos seguirán con su cariño, su amor al niño en duelo, pues ellos son su mundo inmediato. El amor y la protección de sus padres y demás adultos continuará.

9) Recordar que el proceso de duelo y el ajuste a la muerte dura más que los funerales; la depresión más intensa pudiera venir meses después y externalizarse como una falta de atención, una baja en el promedio, soñar despierto, agresión, enuresis, huir de casa o escuela, pesadillas...

10) Tener presente que existen profesionistas que pueden ayudar en el duelo infantil. Buscar ayuda no es un símbolo de aceptar cierta debilidad, pero sí una muestra de seguridad y fuerza propia.

Como en todo, la manera en que se presenta la muerte influye no solamente su concepción tanática, sino también el grado y clase de interés en el tema. Una experiencia traumática, por ejemplo, es insistir que el niño bese el cadáver aunque no quiera; esto puede traer problemas años después.

Dado que todos los niños tienen miedo a quedarse solos o ser abandonados, enseñanzas peligrosas que 
ponen énfasis en quitar al niño de sus padres o castigar a alguno por haberse portado mal, pudiera aumentar el miedo de la muerte hasta convertirse una verdadera obsesión. Como ha señalado Jersild (1954), "señala que la figura de un Dios castigador y un infierno inmisericorde no es la adecuada para ofrecer un consuelo efectivo para los niños".

La manera en que se presenta la muerte al niño, influirá no solamente en sus conceptos sino también en los grados y clases de interés en el tema. Sobre todo, es obvio que muchas preguntas infantiles sobre la muerte son iguales o parecidas a las preguntas de los adultos. Tenemos que concientizarnos de esto y reconocer al niño como un individuo capaz de entender en su nivel. Los niños necesitan y se merecen explicaciones abiertas y honestas. A los que no se permiten pasar el proceso de duelo, tienen tareas incompletas en cuanto a este proceso. Una experiencia exitosa de duelo en la niñez ayuda mucho en duelos futuros. Las reacciones del niño serán comprendidas mejor, si el niño es animado a expresar sus pensamientos y sentimientos. Sólo externando sus dudas y penas, se puede trabajar en su duelo; en esto no existe mucha diferencia entre el niño y el hombre.

\section{Caras vemos, intenciones no sabemos}

\section{A. Fleming-Holland}

¿Cuál clase de dolor hace a la muerte más atractiva que el vivir? En la práctica psicoterapéutica de la autora trabajando con jóvenes, suelen existir temas dolorosos recurrentes. Por ejemplo, la muerte de un ser querido hace recordar la fragilidad de la vida. Se escucha a los jóvenes estar deprimidos por una pérdida, pasada o presente, actual o simbólica; ya no contaban con amigos que habían cambiado a otra ciudad, o quienes ya no fueron amigos, familias cambiadas por el divorcio o la separación, noviazgos terminados, mascotas muertas, la falta de comunicación familiar y la resultante pérdida de la autoestima, y otros temas más pueden tener un impacto poderoso en una persona joven que todavía no tiene los años de experiencia en el vivir para amortiguar el dolor.

Cuando un adolescente se suicida, otros jóvenes pudieran pensar que la muerte es una salida viable de cualquier dolor o dificultad que están enfrentando. Los adolescentes presentan muchos rasgos para la comprensión y aceptación y aún más cuando no las hay en sus casas. Los jóvenes hablan de la inhabilidad de los adultos para entender su mundo; es frecuente que piensen que los adultos ni siquiera intentan comprenderlos. A la vez, expresan su enojo al mundo adulto por no "hacer algo" y al mismo tiempo por lo que ven en la carencia adulta para hacer algo significativo para ayudarles. La inconsistencia suele aparecer en el deseo que sus padres les escuchan, pero al mismo tiempo sienten que el hablar con ellos sería de poca ayuda. Esta autora ha llevado muchas sesiones de terapia familiar en las cuales un joven mostró sorpresa cuando sus padres anunciaron sus deseos de ayudarle; luego comentaron su miedo de que no contaban con una ayuda verdadera de sus familiares ¿Por qué esta contradicción? En parte estriba en que los padres muchas veces se aferran en una visión de qué quieren de su hijo, y qué piensan que debería ser, en vez de intentar ver a su hijo como realmente es.

Los adolescentes hablan mucho de las expectativas de ellos mismos y las que otros tienen de ellos. Las expectativas significan presión emocional, especialmente cuando parecen ser fuera de su alcance, lo cual engendra enojo o desesperación, o ambos. El enojo generalmente se expresa con actitudes o conductas agresivas con los padres y otros adultos que se perciben como controladores en su vida. La desesperación muchas veces conduce a darse por vencido; éstos son los jóvenes que no comparten su dolor con nadie, y quienes sufren una baja en sus calificaciones escolares.

Cuando se siente presión se busca un escape: para el adolescente, este escape usualmente toma la forma de drogas y/o relaciones sexuales. Las drogas (y específicamente el alcohol) se consiguen fácilmente y además es una manera rápida de escaparse del mundo. La inhabilidad de controlar su uso de alcohol o drogas genera más desesperación, pero añadido a sentimientos de culpa. La búsqueda de la comprensión y el amor muchas veces resulta en relaciones sexuales tempranas, con los conocidos resultados desastrosos.

Los adolescentes una y otra vez afirman que quieren más control sobre sus vidas. Unos perciben que los adultos tienen todo el control; otros ven el mundo intelectual fuera de un control adecuado por lo que lo dirigen: los adultos. Los jóvenes pueden hablar de la amenaza de la guerra nuclear y los peligros ambientales con una exactitud no siempre escuchados por boca de un adulto. Aun con los conocimientos técnicos limitados en estas áreas, parecen ser más conscientes que los adultos del enorme daño que se ha desencadenado en el planeta.

Cuando uno se percibe como impotente, a menudo lucha por el control. Esta lucha trae conflictos entre el joven y su familia, maestros y otros adultos. Algunos jóvenes tratan de ganar un sentido de control propio 
por negar que otros tengan el poder sobre él; cuando el alcohol o la droga forman parte del desafío, se presenta una paradoja cruel. El uso de sustancias que alteran la conciencia aparenta establecer más control cuando en realidad se reduce aún más el propio control. Su desafío también trae sensaciones de los adultos que disminuye aún más el control de su propia vida. Empieza un círculo vicioso. Los adultos se ponen duros e imponen restricciones, el joven lucha más por mantener algo de control por sí mismo. Este círculo vicioso a veces resulta en desafío, castigo, y desilusión. Para otros, la lucha resulta más productiva pero aun así dolorosa y difícil; mientras confrontan a sí mismo a los demás tiene que resistir presiones y expectativas externas lo suficiente para que puedan identificar sus propias esperanzas y sueños de vida.

El suicidio de un joven es un grito de ayuda: si se oye, lo que se escucha es jvean mi dolor! Cuando se le pregunta a un joven afligido cómo pudiera ayudar a algún familiar u otra persona, a menudo contesta "sólo escuchar”. Si es un adolescente quien está leyendo esto, por favor intenta entender que "sólo escuchar" su dolor es muy difícil para un adulto. Los adultos quieren hacerla desaparecer, especialmente los padres, que ven el dolor de sus hijos como algo espantoso, un reflejo de una falla de educación familiar. Quieren protegerles o cambiarles para que no se lastimen, aunque no es la solución. Los padres intentan escuchar pero quizás necesitan saber qué es lo que les pasa más de una sola vez, para que realmente se entienda a través de los filtros de protección de la razón y la autoridad.

Si usted es un padre de familia u otro adulto leyendo esto, ya sabe la dificultad en "sólo escuchar". Un refrán contemporáneo dice: "No todo lo que se enfrenta se puede cambiar, pero no se puede cambiar nada hasta que lo enfrente”. Para ayudar a los jóvenes, se necesita reconocer que mucho de lo doloroso en sus vidas no se puede cambiar. Pero el saber que no se puede hacer del todo bien como se quiere, tampoco significa que no se debe intentar escuchar. De todas las lecciones que esta autora ha aprendido de los jóvenes en terapia, quizás la más importante es que el dolor no es siempre malo, porque del dolor crecimos y maduramos. Es parte de la vida que no se puede evitar.

\section{Discusión}

Si protegiéramos las quebradas de los vendavales, nunca veríamos la belleza de sus tallados

KüBLER-Ross
El duelo no es un signo de debilidad o indulgencia a sí mismo, sino una demostración de la profunda necesidad humana que la mayoría tenemos cuando sufrimos la pérdida de un ser querido. No reconoce límites de edad y, además, es multidimensional en el sentido de que se presenta en varias situaciones de pérdida y se manifiesta en múltiples maneras representativas: duelo anticipativo, duelo del sobreviviente (culpabilidad), duelo existencial, duelo del "aniversario" e, incluso, duelo bipolar con labilidad emocional (Feifel, 1989).

Es necesario, como miembros de una sociedad humana, frenar la tendencia actual de "privatizar" el duelo. Hay un proverbio tradicional que dice que "lamentar es sólo sufrir más”. La comunidad necesita agrandar su red institucional y los recursos comunitarios para responder al proceso de duelo en sus miembros. Suprimirlo o minimizarlo, o no reconocer sus aspectos saludables, no es adaptativo para la persona, la familia o la comunidad. Incluso hoy en día empieza a cundir la idea de que cuando la comunidad comparte el duelo de sus miembros, los sentimientos de culpa y depresión de los sobrevivientes se reducen, conjuntamente con el riesgo de una ruptura en el tejido social de la comunidad (Berti y Schneider-Berti, 2003; Feifel, 1989; García-Alandete y Gallego-Pérez, 2003).

Los profesionistas de la salud deben ser precavidos al aconsejar a los sobrevivientes en su duelo: no deben proyectarse sentimientos propios de incomodidad o dolor en los demás que corten su dolor prematuramente, descuiden sus sentimientos dolorosos o, incluso, posterguen su duelo. Si el duelo se descuida o se corta, su expresión pudiera aparecer después de manera inapropiada y regresiva. Aunque el proceso del duelo carece de criterios precisos como entidad clínica, se sabe que el duelo enmascarado puede aparecer en los niños como ausentismo o enuresis, en los adolescentes como farmacodependencia y/o delincuencia, y en los adultos de maneras tan diversas como la promiscuidad, el suicidio y una variedad de enfermedades emocionales y orgánicas. En los últimos 50 años se ha adquirido mucha información referente a grupos de "alto riesgo" entre los sobrevivientes, los que caen en cuadros somáticos y emocionales, especialmente los primeros dos años después de la muerte de un ser querido. No es casual que sea tan alto el porcentaje de los casos en que muere un cónyuge y muere dentro del año siguiente el sobreviviente. El acongojado no solamente experimenta privación sexual, compañía y/o apoyo económico. Además, es vulnerable a la pérdida de su papel social, su autonomía y su sentido de poder (Parkes, 1972). 
Hay tantas maneras diferentes de vivir como maneras de morir y acongojar. A pesar de su popularidad como explicación, dado que ofrece cierta ecuanimidad, los datos científicos no apoyan la existencia de horarios ni etapas fijas que caractericen a los moribundos o al duelo; de igual manera, no apoyan la existencia de la reencarnación o el concepto de un paraíso después de morir. Pero esto no quiere decir que, por ejemplo, las "etapas de morir" de Kübler-Ross (1969) o las "fases del duelo" de Bowlby (1980) no sirvan como orientación sobre las implicaciones y las dinámicas de los procesos de morir y acongojar; son útiles como "instrumentos teóricos”, es decir, más no son acontecimientos inevitables.

Esto requiere una redefinición de las funciones del profesionista de la salud, especialmente del psicoterapeuta. Es necesario hacer caso a la advertencia de Weisman (1975) de que una muerte o duelo "apropiado" es lo que es aceptable a los acongojados, y no lo que los profesionistas de la salud, los familiares o la comunidad llegan a creer.
Por último, el quehacer tanático no es solamente trabajar con los moribundos y sus sobrevivientes directamente, sino preparar a la comunidad global con una amplia y profunda educación tanática como parte del proceso de vivir. Sus formas de expresión pueden variar de individuo a individuo, de cultura a cultura, y pueden diferir según el contexto social y la etapa de desarrollo, pero la presencia psicológica de la muerte siempre está con nosotros. Es demasiado tarde empezar una filosofía de la vida y la muerte cuando se está moribundo o en el proceso de duelo. Como dice Feifel (1977), la pertinencia de una educación sobre la muerte no debería ser solamente para los profesionistas de la salud, los cuales atienden a los moribundos y sus familiares, sino para todas y cada una de las personas. Nuestro mandato como profesionistas de la salud tiene que superar las medidas paliativas para llegar a alterar la conciencia global y producir un cambio sobre las actitudes individuales y culturales sobre la muerte y, por ende, sobre la vida.

\section{Referencias}

Berti, J. A. \& Schneider-Berti, A. E. (2003). Cuando un hijo muere. Logoterapia y ayuda mutua en el proceso de duelo: experiencia de los grupos Renacer. Revista Mexicana de Logoterapia, 10, 21-46.

Bowlby, J. (1980). Loss: Sadness and Depression. New York: Rasie Books.

Feifel, H. (Ed.) (1977). New Meaning of Death. New York: McGraw-Hill.

Feifel, H., \& Branscomb, A. B. (1973). Who's Afraid of Death? Journal of Abnormal Psychology, 81, 82-88.

Feifel, H. \& Nagy, V. T. (1986). Coping with Life-Threat and General Life Conflict: Two Diverse Beasts. Ann Arbor: University of Michigan.

Feifel, H. \& Stack, S. (1989). Coping with Conflict Situations: Middleaged and Elderly Men. Psychological and Aging, 4, 26-33.

Fleming-Holland, A. (1991). La muerte: una perspectiva para los profesionistas de la salud. Psicología y Salud, juliodiciembre, 103-109.

Fleming-Holland, A. (1998). El hombre desde el balcón de la salud mental. Figura-Fondo, 2, 1, 59-64.

Fleming-Holland, A. (2000a). Desarrollo psicológico del niño. Revista Psicología, marzo-abril, 2-12.

Fleming-Holland, A. (2000b). Desarrollo de la autonomía infantil. Revista Psicología, marzo-abril, 13-22.

García-Alandete, J. \& Gallego-Pérez, J. F. (2003). ¿Qué es la muerte? Aproximación al acontecimiento definitiva de la existencia terrena. Revista Mexicana de Logoterapia, 10, 2-20.

Hurlock, E. B. (1956). Child Development. New York: McGrawHill Book Company.

Jersild, A. (1954). Children's Ideals, Morals and Religion. En Child Psychology, (4 ed.), New York: Prentice-Hall.
Kastenbaum, R. \& Costa, P. T. (1977). Psychological Perspectives on Death. Annual Review of Psychology, 28, 255-249.

Kübler-Ross, E. (1969). On Death and Dying. New York: MacMillan.

Kübler-Ross, E. (1982). Working it Through. New York: MacMillan.

Kübler-Ross, E. (1993). Una luz que se apaga. México: Pax.

Markusen, E. \& Fulton, R. (1971). Children Bereavement and Behavior Disorders: A Critical Review, Omega, 2.

Nagy, M. H. (1953). Children's Ideas of the Origin of Illness. Health Education Journal, 9, 6-12.

Parkes, C. M. (1972). Bereavement: Studies of Grief in Adult Life. New York: International Universities Press.

Peterson, C. \& Seligman, M. (2004). Character Strengths and Virtues: A Handbook and Classification. New York: Oxford University Press.

Schilder, P. \& Weschler, D. (1934). The Attitudes of Children Towards Death. Journal of Genetic Psychology, 45, 406-451.

Siegel, D. (1999). The Developing Mind. New York: The Guilford Press.

Siegel, D. (2006). The Mindful Brain. New York: W.W. Norton.

Siegel, D. \& Hartzell, M. (2004). Parenting from the Inside Out: How a Deeper Self-Understanding Can Help you Raise Children who Thrive. New York: Jeremy P. Tarcher/Penguin.

Toynbee, A. (1968). Man's Concern with Death. New York: McGraw-Hill.

Wahl, C. W. (1959). The Fear of Death; en H. Feifel (Ed.), The Meaning of Death. New York: MacGraw-Hill.

Weisman, A. D. (1975). Thanatology; en A. M. Friedman, H. J. Kaplan y B. J. Saddock (Eds.), Comprehensive Textbook of Psychiatry (2a. Ed.). Baltimore: William y Wilkins. 